\title{
Fluid dynamics of millefiori: Mixing ferrofluid with watercolor
}

\author{
Azar Eslam-Panah $\odot,{ }^{*}$ Cooper Kovar $\odot$, Lisa Panczner $\odot$, and Heidi Reuter \\ Penn State University at Berks, Reading, Pennsylvania 19610, USA
}

(Received 31 July 2020; published 12 November 2020)

\begin{abstract}
This paper is associated with a poster winner of a 2019 American Physical Society's Division of Fluid Dynamics (DFD) Milton van Dyke Award for work presented at the DFD Gallery of Fluid Motion. The original poster is available online at the Gallery of Fluid Motion, https://doi.org/10.1103/APS.DFD.2019.GFM.P0034.
\end{abstract}

DOI: 10.1103/PhysRevFluids.5.110510

Millefiori means "thousand flowers" in Italian. By mixing watercolor with ferrofluid interacting on top of a magnet which is about the size of a thumbnail, we can create fascinating pictures to remind us of the millefiori (Fig. 1). Ferrofluids are consisted of ferromagnetic nanoparticles suspended in a carrier fluid and they show interesting behaviors in the presence of a magnetic field [1]. A superficial glance at ferrofluid reveals a black liquid that is made up of iron-oxide $\left(\mathrm{Fe}_{3} \mathrm{O}_{4}\right)$ particles suspended in an oil such as kerosene. The viscosity of the fluid, the tiny size of the particles, and the particles' constant motion keep the solids from settling out. The magnetic particles in ferrofluid are around $10 \mathrm{~nm}$ in size. The iron oxide makes ferrofluid paramagnetic which indicates that the substance itself is not magnetic, but it reacts when near a local magnetic field and repel away from the source [2]. The magnetic reaction in the ferrofluid manifests in the form of pointed peaks or "spikes" of various sizes, heights, and densities, depending on the strength and relative distance of the magnet (Fig. 2). This is because the particles in the fluid are repelled outward by the magnet. The spikes can also form in rows and columns about the ferrofluid dome, or they can form hexagonally, depending on the orientation of the magnetic field lines of the magnet.

Another useful property of ferrofluid is its natural hydrophobia and its density, which are the result of the carrier liquid (kerosene), so when the oily ferrofluid meets water or water-based products, the two remain separated. The ferrofluid's resistance to water-based liquids can be advantageous in medical, electrical, and mechanical applications, but in this study, it is used to create art. The materials used in the setup are Educational Ferrofluid (EFH) Series, petri dishes, magnets, multiple syringes, multiple 30-gauge needles for paints, a 14-gauge needle for ferrofluid, and colorful paints. By placing a few drops of ferrofluid over a magnet, the ferrofluid forms a spiked dome that holds its shape as long as the droplets remain over the magnet (Fig. 2). The amount of ferrofluid was adjusted until a grid of spikes covered the entire dome, which can be seen in Fig. 2(c).

A Nikon D3400 camera, a micro-105-mm lens, a tripod, and a desk lamp were used for the photography. The camera was set to an exposure time of $1 / 120$ of a second, the ISO was set to 200 , and the aperture was set to 3.9 . It is not necessary to have a $105-\mathrm{mm}$ lens, but its ability to keenly focus on small objects was important for our application, since the ferrofluid dome was roughly the size of a thumbnail.

\footnotetext{
*Corresponding author: aue10@psu.edu
}

Published by the American Physical Society under the terms of the Creative Commons Attribution 4.0 International license. Further distribution of this work must maintain attribution to the author(s) and the published article's title, journal citation, and DOI. 


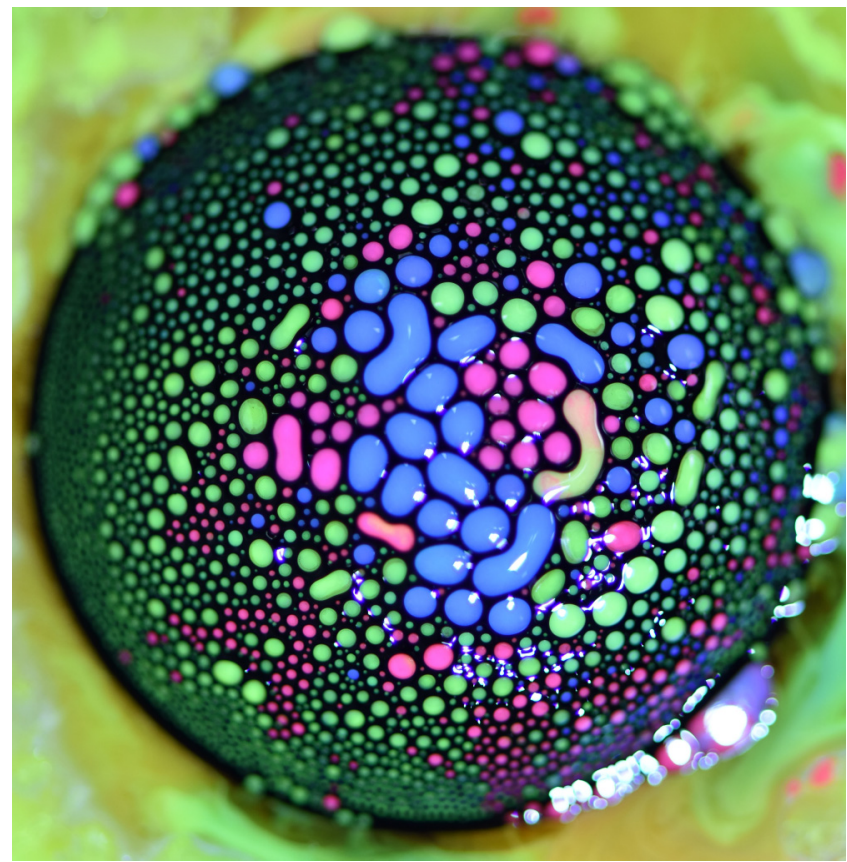

FIG. 1. Mixing ferrofluid with watercolor. For the poster associated to this image see https://doi.org/ 10.1103/APS.DFD.2019.GFM.P0034.

Water-based paint was injected into the dome, just below the surface of the spikes. Because the iron-oxide particles are repelling outward due to the magnet and the water-based paint is lighter than the ferrofluid, the injected paint is pushed outward. Therefore, both fluids (black ferrofluid and colored paint) remain visible as they do not naturally combine with one another and the paint remains at the peak of the dome in more oblong channels [3]. On the other hand, the gravity wanted to pull the paint down, while the magnet was pushing the ferrofluid towards the top. Hence, the paint still created channels within the ferrofluid spikes along the sides and bottom of the dome, but these

(a)

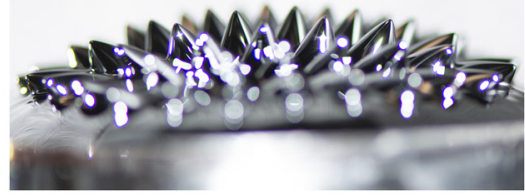

(b)

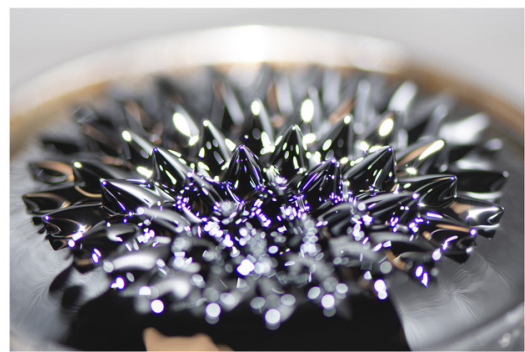

(c)

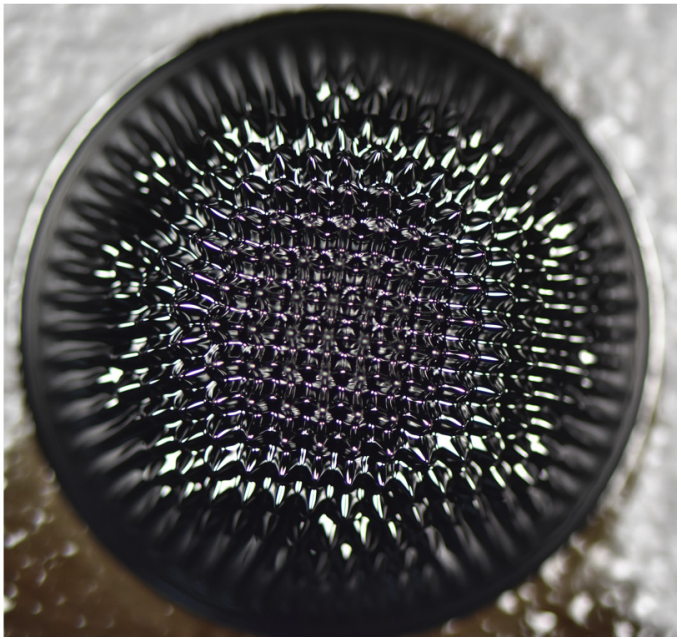

FIG. 2. Ferrofluid dome over magnet before injecting paint: (a) side view, (b) angle view, and (c) top view. 


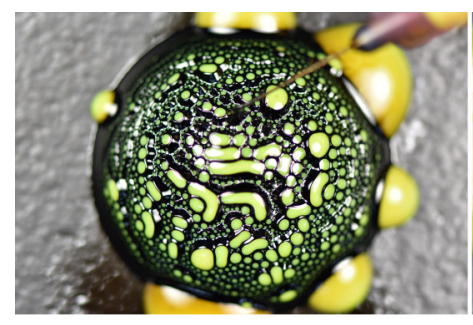

(a)

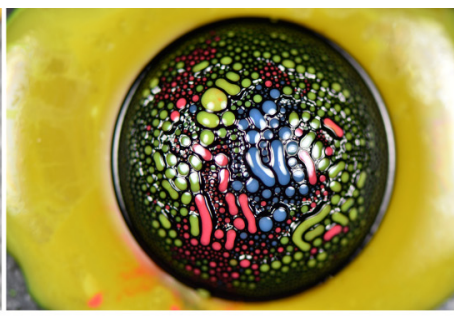

(b)

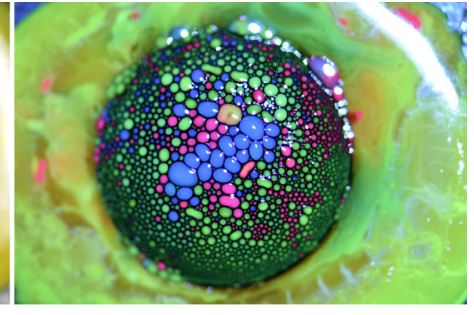

(c)

FIG. 3. Ferrofluid dome over magnet after injecting water-based (a) yellow paint, (b) yellow, red, and blue paints, and (c) mixed color paint.

channels were more like small circular holes. Each color was injected very slowly and carefully right underneath the ferrofluid surface so as to keep the channels intact [Fig. 3(a)]. Over time, the colors around the base of the dome would run and fill the plate surface, which was unintentional but ultimately created the bright and abstract background [Figs. 3(b) and 3(c)].

The authors would like to thank D. Sun for helping with the images which was part of the final project for PHOTO 321N, Flow Visualization, course at Penn State University at Berks. We would also like to acknowledge the support by National Science Foundation with award number CBET-1903312. Moreover, this work was inspired by the work of Swiss photographer, F. Oefner [4].

[1] R. E. Rosensweig, Ferrohydrodynamics (Cambridge University Press, Cambridge, UK, 1985).

[2] J. C. Bacri and D. Salin, Instability of ferrofluid magnetic drops under magnetic field, J. Phys. Lett. 43, 649 (1982).

[3] S. Afkhami, Deformation of a hydrophobic ferrofluid droplet suspended in a viscous medium under uniform magnetic fields, J. Fluid Mech. 663, 358 (2010).

[4] Fabian Oefner blows our mind with these spectacular watercolor and ferrofluid photographs: https://www. itsnicethat.com/articles/fabian-oefner (May 29, 2012). 